\title{
SOLUTION OF AN EXTRAORDINARY DIFFERENTIAL EQUATION BY ADOMIAN DECOMPOSITION METHOD
}

\author{
S. SAHA RAY AND R. K. BERA
}

Received 1 November 2003 and in revised form 18 February 2004

The aim of the present analysis is to apply the Adomian decomposition method for the solution of a fractional differential equation as an alternative method of Laplace transform.

\section{Introduction}

Large classes of linear and nonlinear differential equations, both ordinary as well as partial, can be solved by the Adomian decomposition method $[3,4,6]$. This method is much more simpler in computation and quicker in convergence than any other method available in the open literature.

The application of the fractional differential equation in physical problems is available in the book of Bracewell [11]. Recently, the solution of the fractional differential equation has been obtained through the Adomian decomposition method by the researchers in $[7,14]$.

In this paper, we solve a differential equation containing a fractional derivative of order half along with an ordinary first-order derivative using the Adomian decomposition method. Then the solution obtained by this method is verified with that of the transformed ordinary differential equation derived from the original fractional differential equation.

For the sake of convenience, we first of all give definitions of fractional integral and fractional derivative introduced by Riemann-Liouville as discussed in $[18,20,21]$.

Definition 1.1 (fractional integral). Let $q>0$ denote a real number. Assuming $f(x)$ to be a function of class $C^{(n)}$ (the class of functions with continuous $n$th derivatives), the fractional integral of a function $f$ of order $-q$ is given by

$$
\frac{d^{-q} f(x)}{d x^{-q}}=\frac{1}{\Gamma(q)} \int_{0}^{x} \frac{f(t) d t}{(x-t)^{1-q}}
$$

$d^{-q} f(x) / d x^{-q}$ is also denoted by $\stackrel{x}{I} q_{0}^{q}[7]$.

Copyright (C) 2004 Hindawi Publishing Corporation Journal of Applied Mathematics 2004:4 (2004) 331-338 2000 Mathematics Subject Classification: 44-xx, 26A33 URL: http://dx.doi.org/10.1155/S1110757X04311010 


\section{Solution of an extraordinary differential equation by ADM}

Definition 1.2 (fractional derivative). Let $q>0$ denote a real number and $n$ the smallest integer exceeding $q$ such that $n-q>0(n=0$ if $q<0)$. Assuming $f(x)$ to be a function of class $C^{(n)}$ (the class of functions with continuous $n$th derivatives), the fractional derivative of a function $f$ of order $q$ is given by

$$
\frac{d^{q} f(x)}{d x^{q}}=\frac{d^{n}}{d x^{n}}\left(\frac{d^{-(n-q)} f(x)}{d x^{-(n-q)}}\right)=\frac{1}{\Gamma(n-q)} \frac{d^{n}}{d x^{n}} \int_{0}^{x} \frac{f(t) d t}{(x-t)^{1-n+q}},
$$

$d^{q} f(x) / d x^{q}$ is also denoted by $\stackrel{x}{I}_{0}^{-q} f[7]$.

Definition 1.3 (Mittag-Leffler function). A two-parameter function of the Mittag-Leffler type is defined by the series expansion $[2,21]$

$$
E_{\alpha, \beta}(z)=\sum_{k=0}^{\infty} \frac{z^{k}}{\Gamma(\alpha k+\beta)} \quad(\alpha>0, \beta>0) .
$$

1.1. The decomposition method. We consider an equation in the form

$$
L u+R u+N u=g
$$

where $L$ is an easily or trivially invertible linear operator, $R$ is the remaining linear part, and $N$ represents a nonlinear operator.

The general solution of the given equation is decomposed into the sum

$$
u=\sum_{n=0}^{\infty} u_{n}
$$

where $u_{0}$ is the solution of the linear part.

Our approach will be to write any nonlinear term in terms of the Adomian $A_{n}$ polynomials. It has been derived by Adomian that $N u=\sum_{n=0}^{\infty} A_{n}$, where the $A_{n}$ are special polynomials obtained for the particular nonlinearity $N u=f(u)$ and generated by Adomian $[3,4,5]$. These $A_{n}$ polynomials depend, of course, on the particular nonlinearity.

The $A_{n}$ are given as

$$
\begin{aligned}
& A_{0}=f\left(u_{0}\right), \\
& A_{1}=u_{1}\left(\frac{d}{d u_{0}}\right) f\left(u_{0}\right), \\
& A_{2}=u_{2}\left(\frac{d}{d u_{0}}\right) f\left(u_{0}\right)+\left(\frac{u_{1}^{2}}{2 !}\right)\left(\frac{d^{2}}{d u_{0}^{2}}\right) f\left(u_{0}\right), \\
& A_{3}=u_{3}\left(\frac{d}{d u_{0}}\right) f\left(u_{0}\right)+u_{1} u_{2}\left(\frac{d^{2}}{d u_{0}^{2}}\right) f\left(u_{0}\right)+\left(\frac{u_{1}^{3}}{3 !}\right)\left(\frac{d^{3}}{d u_{0}^{3}}\right) f\left(u_{0}\right),
\end{aligned}
$$


and can be found from the formula (for $n \geq 1$ )

$$
A_{n}=\sum_{\nu=1}^{n} c(\nu, n) f^{(\nu)}\left(u_{0}\right)
$$

where the $c(\nu, n)$ are products (or sums of products) of $\nu$ components of $u$ whose subscripts sum to $n$, divided by the factorial of the number of repeated subscripts [5].

Therefore, the general solution becomes

$$
u=u_{0}-L^{-1} R \sum_{n=0}^{\infty} u_{n}-L^{-1} N u=u_{0}-L^{-1} R \sum_{n=0}^{\infty} u_{n}-L^{-1} \sum_{n=0}^{\infty} A_{n}
$$

where $u_{0}=\phi+L^{-1} g$ and $L \phi=0$.

To identify the terms in $\sum_{n=1}^{\infty} u_{n}$, it has been derived by Adomian that

$$
u_{n+1}=-L^{-1} R u_{n}-L^{-1} A_{n}, \quad n \geq 0 .
$$

From (1.9), we can write $u_{1}=-L^{-1} R u_{0}-L^{-1} A_{0}$. Thus $u_{1}$ can be calculated in terms of the known $u_{0}$.

Now,

$$
\begin{aligned}
& u_{2}=-L^{-1} R u_{1}-L^{-1} A_{1}, \\
& u_{3}=-L^{-1} R u_{2}-L^{-1} A_{2},
\end{aligned}
$$

and so on.

Hence all the terms of $u$ are now calculated and the general solution is obtained as

$$
u=\sum_{n=0}^{\infty} u_{n}
$$

Recently, the Adomian decomposition method was reviewed and a mathematical model of Adomian polynomials was introduced in [1].

\section{Solution of an extraordinary differential equation}

A relationship involving one or more derivatives of an unknown function $f$ with respect to its independent variable $x$ is known as an ordinary differential equation. Similar relationship involving at least one differintegral of noninteger order may be termed as an extraordinary differential equation. Such an equation is solved when an explicit expression for $f$ is exhibited. As with ordinary differential equations, the solutions of extraordinary differential equations often involve integrals and contain arbitrary constants as discussed in [20]. These types of equations are also known as fractional differential equations. The application of extraordinary differential equation is now available in many physical and 


\section{Solution of an extraordinary differential equation by ADM}

technical areas [21]. It can be mentioned here that the simplified fractional-order differential equation appearing in applied problems is of the form

$$
\begin{aligned}
& \frac{d^{\alpha} y(t)}{d t^{\alpha}}+A y(t)=f(t) \quad(t>0), \\
& y^{(k)}(0)=0 \quad(k=0,1, \ldots, n-1),
\end{aligned}
$$

where $n-1<\alpha \leq n$. For $0<\alpha \leq 2$, this equation is called the relaxation-oscillation equation [21]. Moreover, the Bagley-Torvik equation [10]

$$
\begin{gathered}
A \frac{d^{2} y(t)}{d t^{2}}+B \frac{d^{3 / 2} y(t)}{d t^{3 / 2}}+C y(t)=f(t) \quad(t>0), \\
y(0)=0, \quad y^{\prime}(0)=0
\end{gathered}
$$

(where $A \neq 0$ and $B, C \in \mathbb{R}$ ) arises in the modelling of the motion of a rigid plate immersed in a Newtonian fluid [21]. Another equation in the form of

$$
\begin{gathered}
A \frac{d^{2} y(t)}{d t^{2}}+B \frac{d^{1 / 2} y(t)}{d t^{1 / 2}}+C y(t)=f(t), \quad(t>0) \\
y(0)=0, \quad y^{\prime}(0)=0
\end{gathered}
$$

(where $A \neq 0$ and $B, C \in \mathbb{R}$ ) introduced by Caputo [12] arises in the modelling of the motion of a single degree-of-freedom oscillator with damping system [22].

Phenomena in electromagnetics, acoustics, viscoelasticity, electrochemistry, and materials science are described by differential equations of fractional order (see $[8,9,13,15$, $16,17,19])$.

Here we apply the Adomian decomposition method for solving an extraordinary differential equation and then compare this solution with that obtained by an alternative method.

In the present paper, we consider the Adomian decomposition method for solving the following extraordinary differential equation:

$$
\frac{d y}{d x}+\frac{d^{1 / 2} y}{d x^{1 / 2}}-2 y=0
$$

We suppose that $L \equiv d / d x$.

Therefore, by the Adomian decomposition method, we can write

$$
y=c-L^{-1}\left(\frac{d^{1 / 2} y}{d x^{1 / 2}}\right)+2 L^{-1} y
$$

where $c$ is an arbitrary constant. This implies that

$$
y=c-\frac{d^{-1 / 2} y}{d x^{-1 / 2}}+2 \frac{d^{-1} y}{d x^{-1}}
$$


In the light of the Adomian decomposition method, we assume $y(x)=y_{0}(x)+y_{1}(x)+$ $y_{2}(x)+\cdots$ to be the solution of $(2.4)$, where

$$
\begin{gathered}
y_{0}(x)=c, \\
y_{1}(x)=2 \frac{d^{-1} y_{0}(x)}{d x^{-1}}-\frac{d^{-1 / 2} y_{0}(x)}{d x^{-1 / 2}}=\left(2 x-2 \frac{\sqrt{x}}{\sqrt{\pi}}\right) c \\
y_{2}(x)=2 \frac{d^{-1} y_{1}(x)}{d x^{-1}}-\frac{d^{-1 / 2} y_{1}(x)}{d x^{-1 / 2}}=\left(2 x^{2}-\frac{16}{3} \frac{x^{3 / 2}}{\sqrt{\pi}}+x\right) c .
\end{gathered}
$$

Similarly,

$$
\begin{aligned}
& y_{3}(x)=\left(\frac{4}{3} x^{3}-\frac{32}{5} \frac{x^{5 / 2}}{\sqrt{\pi}}+3 x^{2}-\frac{4}{3} \frac{x^{3 / 2}}{\sqrt{\pi}}\right) c, \\
& y_{4}(x)=\left(\frac{2}{3} x^{4}-\frac{512}{105} \frac{x^{7 / 2}}{\sqrt{\pi}}+4 x^{3}-\frac{64}{15} \frac{x^{5 / 2}}{\sqrt{\pi}}+\frac{1}{2} x^{2}\right) c, \\
& y_{5}(x)=\left(\frac{4}{15} x^{5}-\frac{512}{189} \frac{x^{9 / 2}}{\sqrt{\pi}}+\frac{5}{3} x^{3}-\frac{128}{21} \frac{x^{7 / 2}}{\sqrt{\pi}}-\frac{8}{15} \frac{x^{5 / 2}}{\sqrt{\pi}}+\frac{10}{3} x^{4}\right) c,
\end{aligned}
$$

and so on.

Therefore, the solution of (2.4) is

$$
y(x)=c\left(1+3 x+\frac{11}{2} x^{2}-2 \frac{\sqrt{x}}{\sqrt{\pi}}-\frac{20}{3} \frac{x^{3 / 2}}{\sqrt{\pi}}-\frac{56}{5} \frac{x^{5 / 2}}{\sqrt{\pi}}+\cdots\right) .
$$

It can be written as

$$
y(x)=\frac{c}{3}\left(2 \sum_{k=0}^{\infty} \frac{(-1)^{k} 2^{k} x^{k / 2}}{\Gamma(k / 2+1)}+\sum_{k=0}^{\infty} \frac{x^{k / 2}}{\Gamma(k / 2+1)}\right) .
$$

As in $[2,21]$, the explicit formula for $E_{1 / 2,1}(z)$ is

$$
E_{1 / 2,1}(z)=\sum_{k=0}^{\infty} \frac{z^{k}}{\Gamma(k / 2+1)}=e^{z^{2}}(1+\operatorname{erf}(z)) .
$$

That is,

$$
E_{1 / 2,1}(z)=e^{z^{2}} \operatorname{erfc}(-z) .
$$

Therefore, the solution of (2.4) becomes

$$
y(x)=\frac{c}{3}\left(2 e^{4 x} \operatorname{erfc}(2 \sqrt{x})+e^{x} \operatorname{erfc}(-\sqrt{x})\right) .
$$


336 Solution of an extraordinary differential equation by ADM

\section{Verification of the solution}

We can convert (2.4) into an ordinary differential equation.

Applying $d^{-1 / 2} / d x^{-1 / 2}$ to both sides of (2.4), we get

$$
\frac{d^{1 / 2} y}{d x^{1 / 2}}+y-c x^{-1 / 2}-2 \frac{d^{-1 / 2} y}{d x^{-1 / 2}}=0 .
$$

This implies that

$$
2 y-\frac{d y}{d x}+y-c x^{-1 / 2}-2 \frac{d^{-1 / 2} y}{d x^{-1 / 2}}=0 .
$$

After differentiating (3.2), we get

$$
2 \frac{d y}{d x}-\frac{d^{2} y}{d x^{2}}+\frac{d y}{d x}+\frac{c}{2} x^{-3 / 2}-2 \frac{d^{1 / 2} y}{d x^{1 / 2}}=0 .
$$

This implies that

$$
2 \frac{d y}{d x}-\frac{d^{2} y}{d x^{2}}+\frac{d y}{d x}+\frac{c}{2} x^{-3 / 2}-2\left(2 y-\frac{d y}{d x}\right)=0
$$

or

$$
\frac{d^{2} y}{d x^{2}}-5 \frac{d y}{d x}+4 y=\frac{c}{2} x^{-3 / 2}
$$

The solution of (3.5) is

$$
y(x)=c_{1} e^{4 x}+c_{2} e^{x}+\frac{c}{3}\left[-2 e^{4 x}-e^{x}+2 e^{4 x} \operatorname{erfc}(2 \sqrt{x})+e^{x} \operatorname{erfc}(-\sqrt{x})\right],
$$

where $c_{1}, c_{2}$, and $c$ are arbitrary constants.

The function $y(x)$ given in (3.6) will be a solution of (2.4) if $c_{1}=2 c / 3$ and $c_{2}=c / 3$.

Therefore, the solution becomes

$$
\begin{aligned}
y(x) & =\frac{2 c}{3} e^{4 x}+\frac{c}{3} e^{x}+\frac{c}{3}\left[-2 e^{4 x}-e^{x}+2 e^{4 x} \operatorname{erfc}(2 \sqrt{x})+e^{x} \operatorname{erfc}(-\sqrt{x})\right] \\
& =\frac{c}{3}\left[2 e^{4 x} \operatorname{erfc}(2 \sqrt{x})+e^{x} \operatorname{erfc}(-\sqrt{x})\right] .
\end{aligned}
$$

Solution (3.7) completely matches solution (2.13) obtained by the Adomian method.

\section{Conclusion}

It is observed that although the extraordinary differential equation can be converted into an ordinary differential equation, the way of solving that ordinary differential equation is quite laborious and requires enough skill. Moreover, an extraordinary differential equation can also be solved by Laplace transform method. But sometimes it becomes 
cumbersome to have the solution because of finding out its inverse. In order to avoid this, we can use the Adomian decomposition method, which gives a quite satisfactory result.

\section{Acknowledgment}

We express our sincere thanks to the referee for his kind comments for the improvement of the paper.

\section{References}

[1] F. Abdelwahid, A mathematical model of Adomian polynomials, Appl. Math. Comput. 141 (2003), no. 2-3, 447-453.

[2] M. Abramowitz and I. A. Stegun, Handbook of Mathematical Functions with Formulas, Graphs, and Mathematical Tables, National Bureau of Standards Applied Mathematics Series, vol. 55, U.S. Government Printing Office, District of Columbia, 1964.

[3] G. Adomian, Nonlinear Stochastic Operator Equations, Academic Press, Florida, 1986.

[4] Nonlinear Stochastic Systems Theory and Applications to Physics, Mathematics and Its Applications, vol. 46, Kluwer Academic Publishers Group, Dordrecht, 1989.

[5] Solving Frontier Problems of Physics: The Decomposition Method, Fundamental Theories of Physics, vol. 60, Kluwer Academic Publishers Group, Dordrecht, 1994.

[6] G. Adomian and R. Rach, On linear and nonlinear integro-differential equations, J. Math. Anal. Appl. 113 (1986), no. 1, 199-201.

[7] H. L. Arora and F. I. Abdelwahid, Solution of non-integer order differential equations via the Adomian decomposition method, Appl. Math. Lett. 6 (1993), no. 1, 21-23.

[8] Y. Babenko, Non integer differential equation, International Conference Bordeaux, Bordeaux, 1994.

[9] _ Non integer differential equation in engineering: chemical engineering, International Conference Bordeaux, Bordeaux, 1994.

[10] R. L. Bagley and P. J. Torvik, On the appearance of the fractional derivative in the behavior of real materials, Trans. ASME J. Appl. Mech. 51 (1984), 294-298.

[11] R. N. Bracewell, The Fourier Transform and Its Applications, McGraw-Hill, New York, 1978.

[12] M. Caputo, Elasticità e Dissipazione, Zanichelli, Bologna, 1969.

[13] L. Gaul, P. Klein, and S. Kempfle, Damping description involving fractional operators, Mech. Systems Signal Processing 5 (1991), 81-88.

[14] A. J. George and A. Chakrabarti, The Adomian method applied to some extraordinary differential equations, Appl. Math. Lett. 8 (1995), no. 3, 91-97.

[15] W. G. Glockle and T. F. Nonnenmacher, Fractional integral-operators and fox functions in the theory of viscoelasticity, Macromolecules 24 (1991), 6426-6434.

[16] F. Mainardi, Fractional relaxation and fractional diffusion equations, mathematical aspects, Proceedings of the 14th IMACS World Congress on Computation and Applied Mathematics Vol. 1 (Atlanta), 1994, pp. 329-332.

[17] M. W. Michalski, Derivatives of noninteger order and their applications, Dissertationes Math. (Rozprawy Mat.) 328 (1993), 1-47.

[18] K. S. Miller and B. Ross, An Introduction to the Fractional Calculus and Fractional Differential Equations, John Wiley \& Sons, New York, 1993.

[19] M. Ochmann and S. Makarov, Representation of the absorption of nonlinear waves by fractional derivatives, J. Acoust. Soc. Amer. 94 (1993), no. 6, 3392-3399.

[20] K. B. Oldham and J. Spanier, The Fractional Calculus, Mathematics in Science and Engineering, vol. 111, Academic Press, New York, 1974. 


\section{Solution of an extraordinary differential equation by ADM}

[21] I. Podlubny, Fractional Differential Equations, Mathematics in Science and Engineering, vol. 198, Academic Press, San Diego, 1999.

[22] L. E. Suarez and A. Shokooh, An eigenvector expansion method for the solution of motion containing fractional derivatives, Trans. ASME J. Appl. Mech. 64 (1997), no. 3, 629-635.

S. Saha Ray: B. P. Poddar Institute of Management and Technology, Poddar Vihar, 137 VIP Road, Kolkata 700052, India

E-mail address: santanusaharay@yahoo.com

R. K. Bera: Heritage Institute of Technology, Chowbaga Road, Anandapur, Kolkata 700107, India

E-mail address: rasajit@hotmail.com 


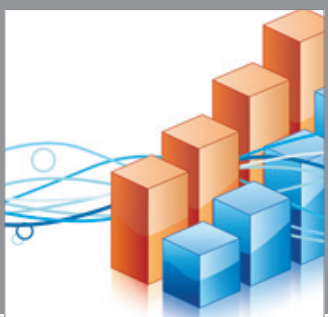

Advances in

Operations Research

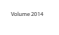

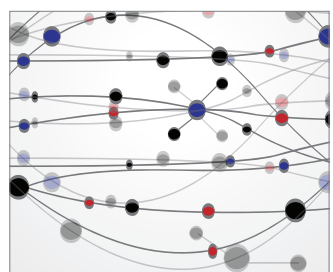

\section{The Scientific} World Journal
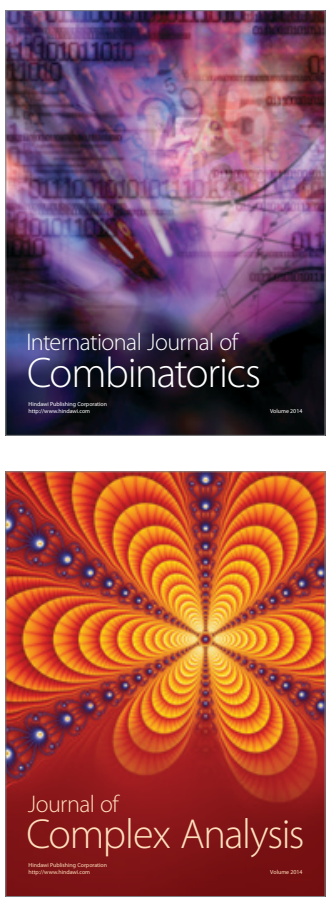

International Journal of

Mathematics and

Mathematical

Sciences
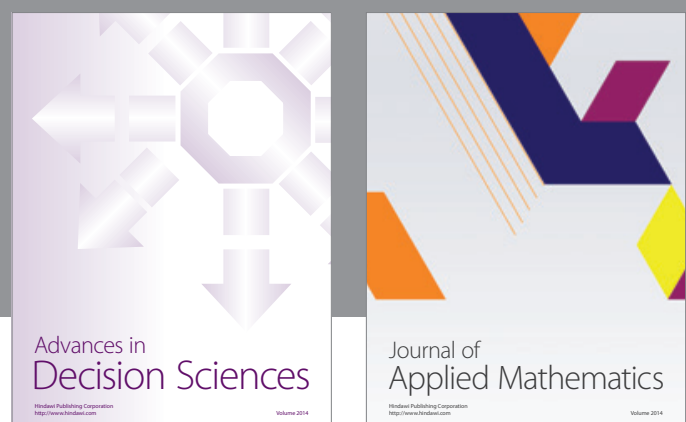

Journal of

Applied Mathematics
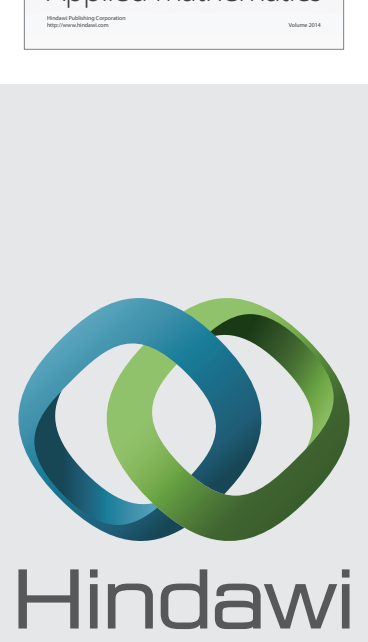

Submit your manuscripts at http://www.hindawi.com
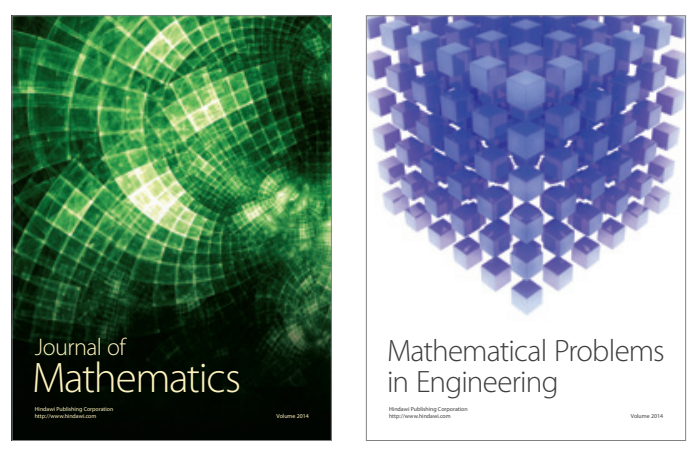

Mathematical Problems in Engineering
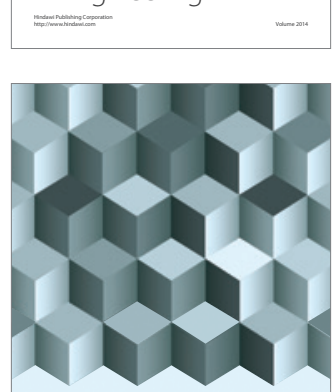

Journal of

Function Spaces
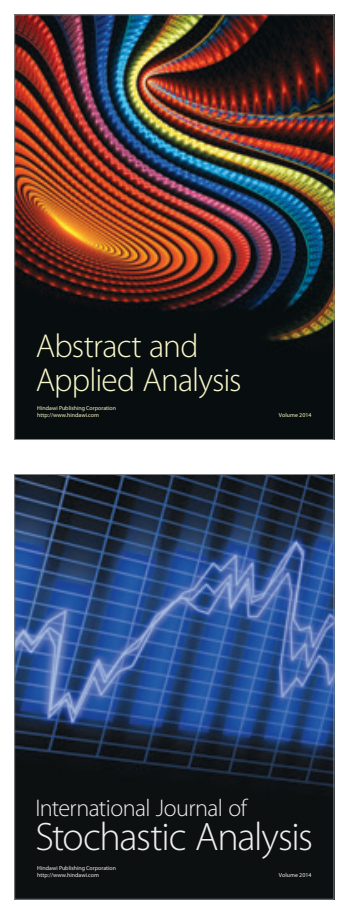

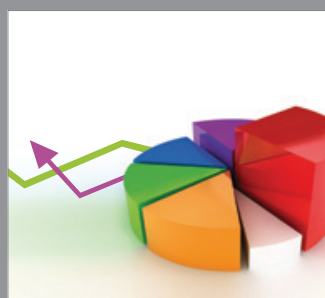

ournal of

Probability and Statistics

Promensencen
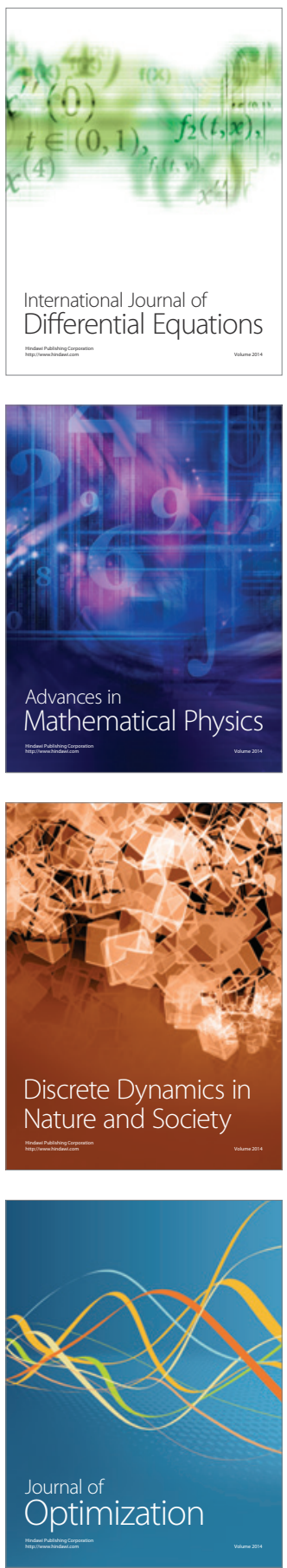